\title{
Madelung disease
}

\author{
Bogna Zielińska-Kaźmierska ${ }^{1,2}$, Michał Lewicki $^{2}$, Bogusława Manowska $^{1}$
}

${ }^{1}$ Clinic of Cranio-Maxillofacial and Oncological Surgery, Medical University of Lodz, Lodz, Poland Head of the Department: Prof. Piotr Arkuszewski MD, PhD

${ }^{2}$ Department of Cranio-Maxillofacial and Oncological Surgery, Norbert Barlicki Memorial Teaching Hospital No. 1, Lodz, Poland Head of the Department: Bogna Zielińska-Kaźmierska MD, PhD

Madelung disease, also known as multiple symmetric lipomatosis (MLS), benign symmetric lipomatosis (BSL) or Launois-Bensaude syndrome, is a rare metabolic condition characterized by symmetric, non-encapsulated (unlike lipomas) fat deposits on the patient's body, usually around the neck, but in some cases also around shoulders, the upper trunk, chest and limbs. It was first described by Benjamin Brody in 1846. In 1888, Otto Madelung presented 35 cases of this disease. In 1898, Launois and Bensaude described another 30 cases of patients with excessive adipose tissue growth around the neck, nape, back and shoulders. This new disease was called Madelung disease or Launois-Bensaude syndrome. From then to the end of 2002, there were around 300 reports presenting cases of patients with this disease in the medical literature $[1,2]$. Launois-Bensaude syndrome may cause breathing difficulties and problems in everyday functioning as it progresses, therefore it requires starting the treatment. However, the most common reasons why the patients decide to undergo treatment isaesthetics and the worsening perception of themselves [3, 4].

Multiple symmetric lipomatosis most often affects white middle-aged men from the Mediterranean population, who have a history of alcohol abuse [3, 5]. Depending on the disease type, fat deposits can be located around the neck and nape, in the shape of a horse collar; parotideomasseteric areas, resembling hamster's cheek pouches; around shoulders, the back and chest giving the patient's body a pseudo-athletic appearance [1, 4-6]. The disease is characterized by the creation of new fat cells (like in the case of pregnant women or infants) instead of an increase in the size of the existing ones [4, 7]. It is not yet fully understood what causes the disease. Sixtyninety percent of patients abuse alcohol and suffer from secondary cirrhosis [8]. There is a theory which suggests that alcohol can influence physiological enzymatic process disorders in mitochondria. Alcohol excess impairs adrenergic lipolysis and may lead to uncontrolled emergence of fat deposits in different parts of the body [6]. It is important to notice that discontinuation of alcohol consumption has no effect on further disease progress [9].

Madelung disease is often accompanied by hypothyroidism, diabetes, megalocytic anemia, cirrhosis caused by alcohol abuse, epilepsy, or polyneuropathies connected with alcohol-related demyelination [1, 5]. In differential diagnosis the following should be considered: Cushing disease, simple obesity, neck cysts, salivary gland and thyroid gland diseases, leukemia and soft-tissue sarcoma [10].

Indications for clinic treatment include breathing difficulties, dysphagia, dysphonia and decreased head movement. According to many authors, despite the presence of the above mentioned symptoms, some patients decide to undergo treatment for aesthetic reasons. Deformations of the visible body parts worsen patients' perception of themselves [11], leading to depressive disorders.

Many authors propose multistage dissection [4, 10], liposuction or lipolysis [12] as a method of treatment. Some reports suggest that pharmacological treatment is effective - there has been an improvement in patients treated with magnesium and vitamin $B_{6}$ [13]. However, the most common and the most effective method is surgical removing the lesion [10]. Big tumors are normally removed at various stages - it depends on the size of the tumor, coagulation problems and impaired wound healing caused by cirrhosis. What is more, vascularization of the lipoma makes it difficult to keep hemostasis during the surgery. Liposuction is the least traumatic procedure, however, it involves the risk of surgical mishap and disease recurrence [14], and therefore it is suggested as a complementary therapy. Dietary treatment is inefficient since lipomas do not disappear even with cancer cachexia. 
Because of the rarity of this disease as well as the controversies connected with its treatment, it seems interesting to present the case of our patient.

The patient, a 46-year-old male, was referred to the Maxillofacial Clinic at Norbert Barlicki Memorial Teaching Hospital No. 1 for diagnosis and treatment of his painless, tender connective tissue tumor that had been growing for the last 3 years and that covered all the front and lateral area of the neck and posterior cervical region (Figure 1). According to the patient's medical history, he was diagnosed with alcohol dependence syndrome and as a consequence, also with alcoholic cirrhosis. The laboratory tests showed hypercholesterolemia, hyperuricemia and hyperbilirubinemia, as well as other concurrent diseases: arterial hypertension and diabetes. The patient's TSH level was low. The neck ultrasound showed general enlargement of salivary glands, and the NMR test (Figure 2) revealed the existence of enlarged salivary glands overgrown with adipose tissue. In order to determine the nature of the lesion, the thin needle biopsy test was run, which demonstrated the chronic inflammatory condition of the connective tissue and adipose tissue. The image seemed to point out to a benign lesion.
The patient was feeling well, he did not report any breathing or food ingestion problems. The lesion did not make his head movement difficult, either. The main reason why the patient decided to undergo treatment was his low self-esteem because of the progressive change in the appearance caused by the disease. It was also the

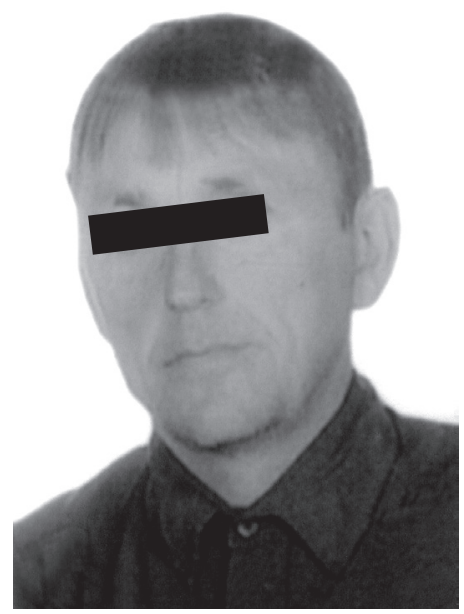

Figure 1. The photo presents the patient before the appearance of the lipoma lesions
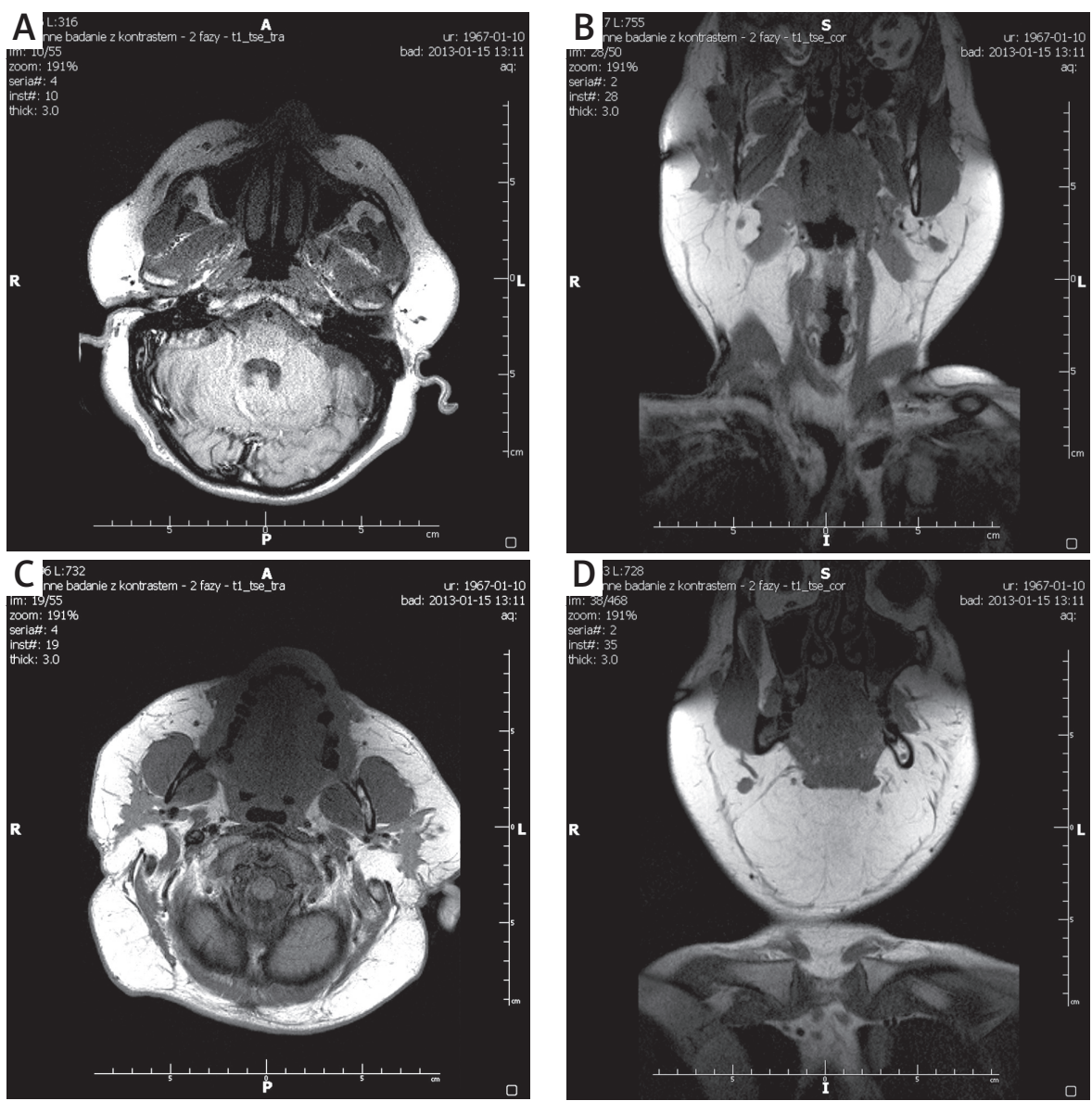

Figure 2 A-D. Magnetic resonance imaging presenting the expansion of the adipose tissue 


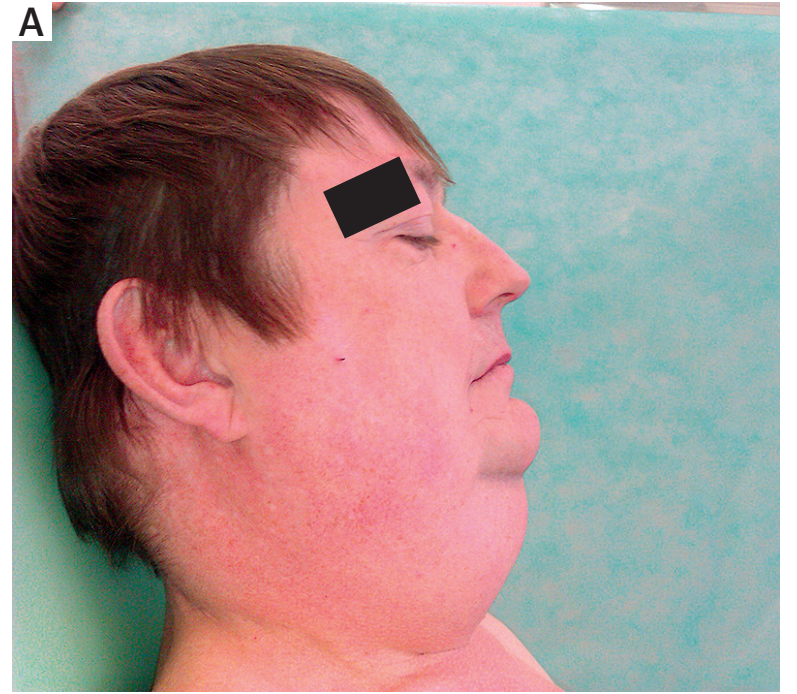

Figure 3 A, B. The pictures present the patient before the first procedure. The front view and the side view present the expansion of the lipoma lesion limited to the face and neck area

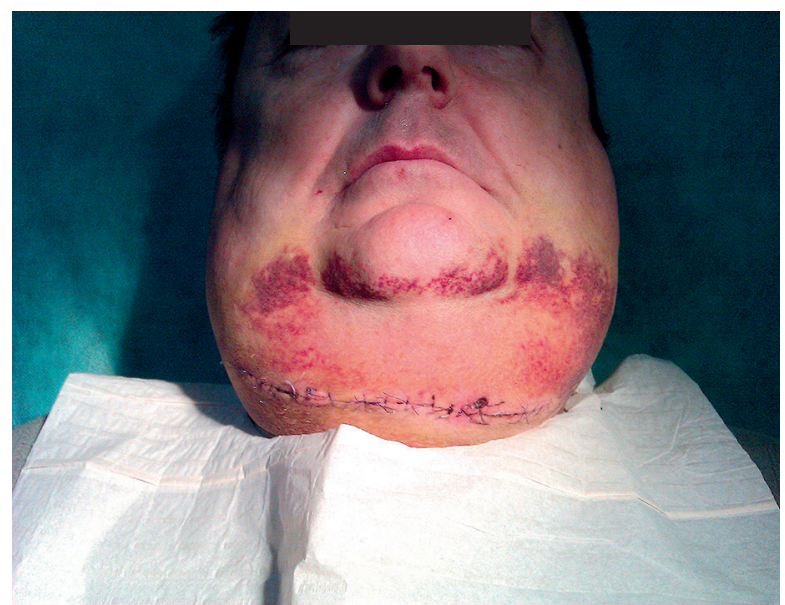

Figure 4 . The patient $24 \mathrm{~h}$ after the first surgery

main factor qualifying the patient for a surgery (Figures $3 \mathrm{~A}, \mathrm{~B})$.

The patient was qualified for a multistage removal of the excess fat tissue from the front and side neck areas. The first procedure was conducted in April 2013. The first stage of the treatment involved removal of excess fat tissue from the front neck area. After stabilizing blood pressure, sugar level and level of liver enzymes, the surgery started. A horizontal section, $4 \mathrm{~cm}$ below the lower edge of the mandible, reached the lipoma lesion, which was then exfoliated en bloc. No connective tissue capsule surrounding the lesion was found. The removed adipose tis-

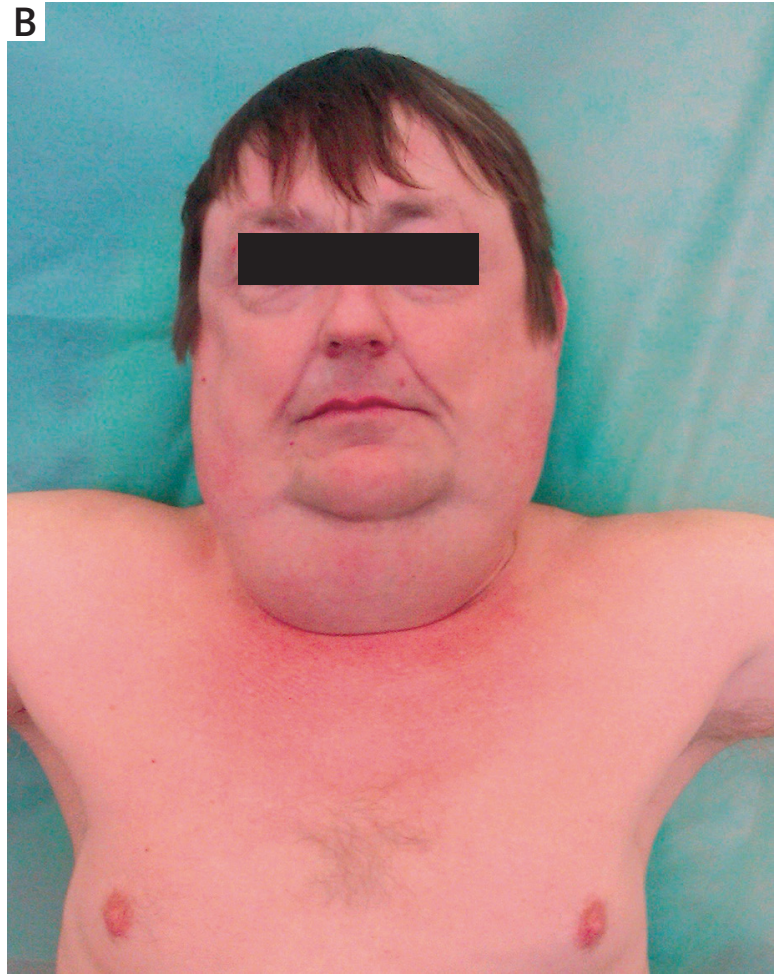

sue was well-vascularized, which made it difficult to keep full hemostasis during the surgery. After removing the lesion, the wound was closed in layers and the Redon drain was placed. Nevertheless, after the surgery a hematoma was observed around the area where the surgery took place (Figure 4). Five days after the surgery, the Redon drain was removed, and after 2 weeks the patient was discharged from hospital.

One year later, the second stage of the procedure took place, which was intended to reduce the lipoma lesion at the request of the patient. However, an increase in size of the lesions around the areas where the surgery was not done (cheeks, nape) was observed, compared to the last time when the patient was hospitalized (Figure 5).

This stage was intended to reduce the adipose tissue around the lateral neck area. Two sections around the lateral neck area in extension of the scar after the first surgery reached the lipoma lesion and the excess of fat was removed. Like during the first surgery, there was a problem with keeping full hemostasis during the procedure. After the surgery, surgical drains were placed on both sides. What is more, a collection of around $50 \mathrm{ml}$ of liquid with traces of blood every $24 \mathrm{~h}$ was revealed, which was progressively decreasing. One of the surgical drains became clogged, which caused a big hematoma on one side of the treated area and wound disruption on the other side. After removing the clots, the wound was prepared for suturing and a new surgical drain was placed. The drain was removed after 5 days. After another 2 days the patient was discharged from the ward 

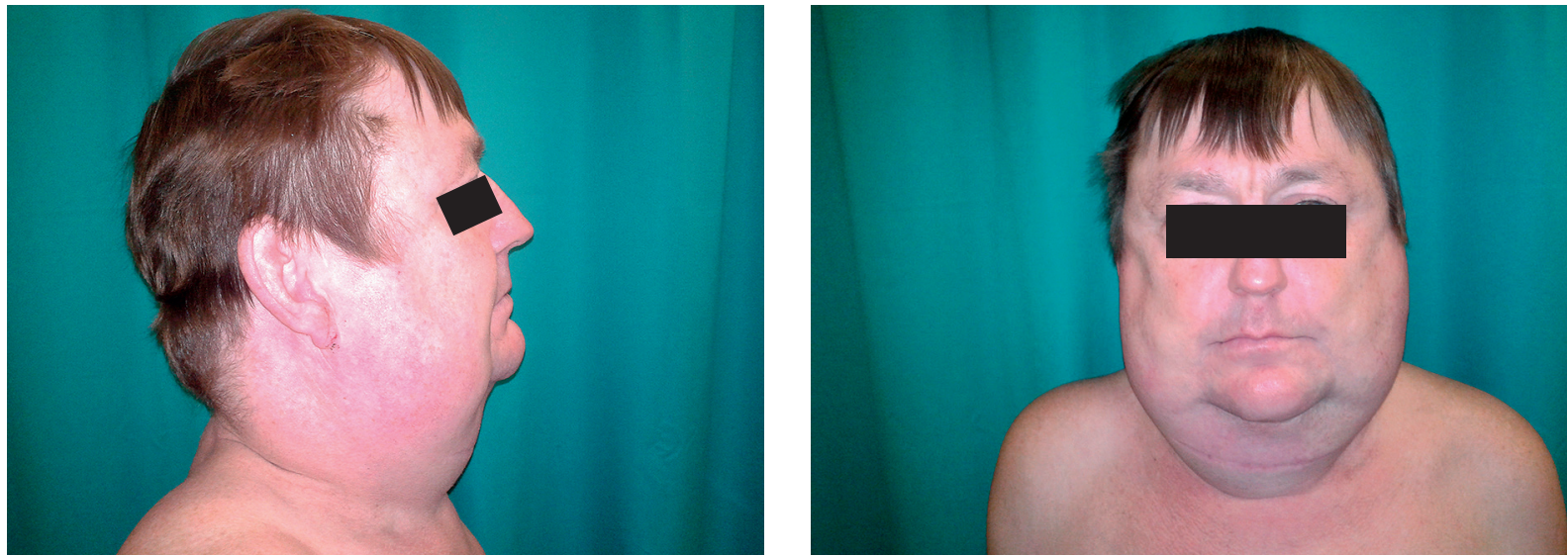

Figure 5. Front view and side view of the patient before the second procedure

and referred to further ambulatory treatment. Until now, the patient has undergone two procedures reducing the amount of adipose tissue which improved his aesthetics. Nevertheless, all the lesion could not be removed, therefore the patient was qualified for the next stage of surgical treatment.

The effects of the conducted surgeries were positively received by the patient despite the complication following the procedure. The surgeries showed what problems may be encountered during the treatment. The patients need careful diagnosis before the surgery and close monitoring after it.

Patients with MSL should be examined taking into consideration the complexity of their problem. The basic factors qualifying a patient for a surgery should be the respiratory and digestive system functioning disorders, as well as problems in everyday functioning. The patient's emotional sphere and his self-perception should not be neglected [10]. Our patient's example shows that despite problems in everyday functioning he was seeking improvement in his appearance and therefore improvement in his self-perception. It should be emphasized that apart from treatment for Madelung disease the patient was also dealing with alcohol abuse.

Moreover, it should be noticed that about $90 \%$ of patients with Madelung disease also have cirrhosis. This is an additional obstacle during the surgical treatment. In spite of the accurate preparation of the patients for the surgery and putting right the abnormalities found in laboratory tests, difficulties with keeping full hemostasis during the surgery occur. The wound healing process after the procedure is also impaired. However, taking into consideration the literature and our own experience, we reach a conclusion that the surgical removal of lipoma lesions seems to be the best and the most effective method.

\section{Conflict of interest}

The authors declare no conflict of interest.

\section{References}

1. Musialik K, Bogdański P, Nawrocka M. Choroba Madelunga - opis przypadku i przegląd piśmiennictwa. Forum Zaburzeń Metabolicznych 2012; 3: 147-53.

2. Bergler-Czop B, Wcisło-Dziadecka D, Brzezińska-Wcisło L. Madelung's disease in a patient with chronic renal insufficiency: a case report and review of literature. Postep Derm Alergol 2014; 31: 121-4.

3. Parmar SC, Blackburn C. Madelung's disease: an uncommon disorder of unknown aetiology? Br J Oral Maxillofac Surg 1996; 34: 467-70.

4. Zhang WJ, Jiang H, Zhang JL, et al. Surgical treatment of multiple symmetric lipomatosis (Madelung's disease): a single center experience. J Oral Maxillofacial Surg 2011; 69: 2448-51.

5. Morinaka S, Sato T, Miyoshi H, et al. A case of multiple symmetrical lipomatosis (Madelung's disease). Auris Nasus Larynx 1999; 26: 349-53.

6. Zancanaro C, Sbarbati A, Morroni M, et al. Multiple symmetric lipomatosis. Ultrastructural investigation of the tissue and preadipocytes in primary culture. Lab Invest 1990; 63: 253-8.

7. Nisoli E, Regianini L, Briscini L, et al. Multiple symmetric lipomatosis may be the consequence of defective noradrenergic modulation of proliferation and differentiation of brown fat cells. J Pathol 2002; 198: 378-87.

8. Meningaud JP, Pitak-Arnnop P, Bertrand JC. Multiple symmetrical lipomatosis: case report a review of the literature. J Oral Maxillofacial Surg 2007; 65: 1365-9.

9. Brackenbury ET, Morgan WE. Surgical management of Launois-Bensaude syndrome. Thorax 1997; 52: 834-5.

10. Zuber M, Pittasch D. Benign symmetric lipomatosis (Launois-Bensaude syndrome) - a rare cause of muscular weakness. Eur J Med Res 2006; 11: 174-7.

11. Josephson GD, Sclafani AP, Stern J. Benign symmetric lipomatosis (Madelung's disease). Otolaryngol Head Neck Surg 1996; 115: 170-1.

12. Iljin A, Kruk-Jeromin J. Lipomatosis. Postep Derm Alergol 2009; 26: 74-8.

13. Dabrowska A, Tarnowska C, Jałowinski R, et al. Multiple symmetric lipomatosis in the otolaryngology as diagnostic and therapeutic problem. Otolaryngol Pol 2005; 59: 717-22.

14. Faga A, Valdatta LA, Thione A, et al. Ultrasound assisted liposuction for the palliative treatment of Madelung's disease: a case report. Aesthetic Plast Surg 2001; 25: 181-3. 